\section{Visual outcomes following Artisan aphakia iris claw lens implantation}

KS Lett and PR Chaudhuri

\begin{abstract}
Objective To assess the visual outcome of eyes undergoing Artisan lens implantation for aphakia, to identify reasons for poor outcomes, and to report incidences of post-operative uveitis, glaucoma, or hyphaema. Methods A retrospective analysis of 32 eyes rendered aphakic through various causes, which subsequently underwent Artisan lens implantation.

Results A total of 32 eyes from 31 patients were reviewed. Follow-up ranged from 6 to 42 months, mean 17.3 months. The commonest cause of aphakia was following complicated phacoemulsification in $\mathbf{1 7}$ of the 32 eyes (53.1\%). At final follow-up, 21 of the 32 eyes $(65.6 \%)$ achieved best-corrected visual acuity (BCVA) better than that measured pre-operatively and 10 of the 32 eyes (31.3\%) matched their pre-operative BCVA. Of 33 eyes, only $1(0.03 \%)$ attained a final BCVA worse than pre-operatively, owing to non-arteritic anterior ischaemic optic neuropathy. A total of 4 of the 33 eyes $(12.5 \%)$ had pre-existing glaucoma or ocular hypertension; no additional intraocular pressure problems were identified during the follow-up period. Conclusion This study suggests that Artisan iris claw lens insertion is beneficial in acquired aphakia, matching or exceeding pre-operative BCVA in the overwhelming majority of the cases. In addition, this series did not identify any post-operative problems with uveitis, glaucoma, or hyphaema. Eye (2011) 25, 73-76; doi:10.1038/eye.2010.146; published online 15 October 2010
\end{abstract}

Keywords: lens; aphakia; intra-ocular; implant

\section{Introduction}

Posterior chamber intracapsular intraocular lens (IOL) implantation remains the ideal outcome following cataract extraction. However, following complicated cataract surgery, predominantly after posterior capsule rupture with or without displacement of nuclear fragments, there may be insufficient remaining capsular support for either intracapsular or posterior chamber sulcus placement of the IOL. A similar scenario is also frequently encountered following pars plana vitrectomy for retrieval of displaced nuclear fragments and congenital subluxated lenses.

In such cases, alternative methods of IOL fixation must be considered. These include sutured scleral fixation, anterior chamber sulcus placement and iris fixation.

Previously, iris fixation was achieved using closed-loop anterior chamber IOLs, with the loops being positioned anterior and posterior to the iris. Pupil dilation carried the risk of IOL displacement and this type of lens fell out of favour in the mid 1980s following numerous reports of significant complications in the post-implantation period. ${ }^{1}$

An alternative method of iris fixation involves claw shaped haptics attached to the mid-peripheral iris. This method of fixation also confers the benefit of easy pupil dilation.

We describe our experience with the Artisan aphakia iris claw lens (Ophtec, Groningen, the Netherlands).

\section{Patients and methods}

All cases of Artisan lens implantation performed over a four-year period at Leicester Royal Infirmary were identified from the operating theatre logbook. In total, 43 patients were identified, of which 38 sets of case notes were retrievable detailing Artisan implantation in 39 eyes. From these, pre- and post-operative data were retrieved, including demographics, pre-existent ocular comorbidity and postoperative complications. The aetiology of
Department of Ophthalmology, University Hospitals of Leicester, Infirmary Square, Leicester, UK

Correspondence: K Lett, Department of Ophthalmology, University Hospitals of Leicester, Leicester Royal Infirmary, Infirmary Square, Leicester, Leicestershire LE1 5WW, UK Tel: + 44 (0)116 258 6478; Fax: + 44 (0)116 2585927 E-mail: kslett@ ophth.freeserve.co.uk

Received: 12 May 2010 Accepted in revised form: 25 August 2010 Published online: 15 October 2010

This data has previously been presented at the Royal College of Ophthalmologists Annual Congress 2008. 
aphakia was identified in each case. Best-recorded visual acuity was noted before and after IOL implantation.

All procedures were performed by the same consultant surgeon using a standardised technique that, in summary, comprised: a $5.2 \mathrm{~mm}$ chord length halfthickness limbal section, creation of two small corneal paracenteses at 2 and 10 o'clock, followed by full thickness completion of the superior corneal incision; injecting intracameral acetylcholine chloride $1 \%$, then cohesive viscoelastic into the anterior chamber (AC); inserting the lens implant vertically into the $\mathrm{AC}$; rotating the IOL such that the claw haptics are orientated dependant on the amount of iris tissue present; enclavation of the iris between the claws; creation of a superior peripheral iridotomy; suturing the large corneal wound; and washing out the AC.

In some patients, pars plana or anterior vitrectomy was required before lens insertion, in which case the pupil was dilated pre-operatively and intracamerally. Miochol was injected following vitreous clearance.

\section{Results}

In total, 39 eyes in 38 patients were analysed. In total, 19 patients were male and 20 were female. Their ages ranged from 22 to 98 years, mean 62.5 years.

Follow-up ranged from 3 to 36 months, mean 15.7 months.

The aetiology of (effective) aphakia is detailed in Table 1. A total of six patients had pre-existing glaucoma or ocular hypertension. Other pre-existing ocular pathology is outlined in Table 2 .

Of the 39 eyes, $26(66.6 \%)$ achieved a final visual acuity better than the pre-operative best recorded visual acuity.

Table 1 Aetiology of aphakia

\begin{tabular}{lc}
\hline Aetiology of aphakia & No. of cases \\
\hline Complicated phaco for senile cataract & 20 \\
Penetrating eye injury/trauma & 9 \\
Congenital cataract extraction & 4 \\
IOL dislocation & 4 \\
Previous ICCE & 2
\end{tabular}

Table 2 Pre-existing ocular comorbidity

\begin{tabular}{lc}
\hline Pre-existing pathology & No. of cases \\
\hline Congenital cataract and amblyopia & 5 \\
Macular degeneration & 3 \\
Previous retinal detachment repair & 1 \\
Coloboma & 1 \\
Epiretinal membrane & 1 \\
Traumatic macular scar & 1 \\
\hline
\end{tabular}

A total of 12 eyes (30.8\%) achieved a final acuity equal to that measured pre-operatively, and only 1 eye $(2.6 \%)$ ended up with a poorer final acuity (see Figure 1). This patient had Marfan's syndrome and was diagnosed with non-arteritic anterior ischaemic neuropathy shortly after Artisan implantation. It is possible that this developed during the course of general anaesthesia for the procedure. The majority of patients were operated on under local anaesthesia and all were elective procedures.

Of the 39 eyes, $25(64.1 \%)$ achieved a post-operative visual acuity equal to or better than $6 / 12$. Of these, two eyes had ocular co-pathology. One eye was amblyopic and had undergone congenital cataract extraction; the other eye had dry macular degeneration. A total of 14 eyes $(35.9 \%)$ achieved final visual acuities worse than 6/12, of which 12 eyes $(85.7 \%$ ) contained ocular co-pathology as previously detailed (see Table 3 ).

Apart from the patient with Marfan's syndrome described above, no other patient developed any additional pathology following Artisan implantation. There were no intra-operative complications in this case series. No cases of uveitis, hyphaema, or post-operative glaucoma were identified in any patient. No cases of post-operative hypotony were reported —all cases measured intra-ocular pressures within the normal range at final follow-up. No cases of cystoid macular oedema or corneal decompensation were seen.

\section{Discussion}

The aim of Artisan aphakia lens implantation is to achieve acceptable refractive and visual outcomes in patients with insufficient capsular support for intracapsular or sulcus IOL placement, while avoiding the need for aphakic spectacles or contact lenses. It is a vaulted convex-concave design $-5 \mathrm{~mm}$ optic with an overall diameter of $8.5 \mathrm{~mm}$, including the haptics. The haptics are designed as pincers or claws, which grasp the immobile part of the peripheral iris thus avoiding the difficulties in pupil dilation experienced with iris clip lenses and enable excellent fundal visualisation.

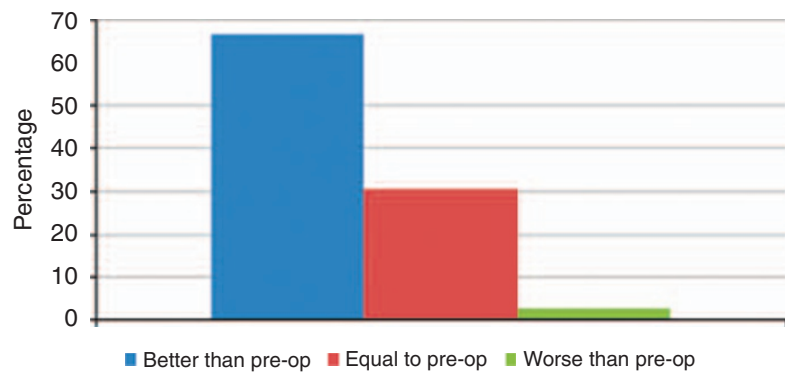

Figure 1 Pre-operative $v s$ final VA. 
Table 3 Patients with final VA worse than $6 / 12$

\begin{tabular}{lccll}
\hline Pt. age & Pre-op VA & Post-op VA & Comorbidity & Aetiology of aphakia \\
\hline 91 & $6 / 60$ & $6 / 60$ & Macular degeneration & ICCE \\
23 & $6 / 36$ & $6 / 18$ & Amblyopia & Cong. Cat. Extraction \\
38 & $6 / 18$ & $3 / 18$ & Marfan's syndrome, post-op NAAION & IOL dislocation \\
55 & HM & HM & Previous RRD repair & Complicated phaco \\
33 & $6 / 18$ & $6 / 18$ & Amblyopia & Cong. Cat. Extraction \\
40 & $6 / 60$ & $6 / 60$ & Penetrating eye injury & Macular scar \\
86 & $\mathrm{CF}$ & $6 / 18$ & None & Complicated phaco \\
22 & $6 / 24$ & $6 / 24$ & Amblyopia & Cong. Cat. Extraction \\
98 & $\mathrm{CF}$ & $3 / 24$ & Macular degeneration & Complicated phaco \\
52 & $1 / 24$ & $1 / 12$ & Coloboma & Complicated phaco \\
48 & $6 / 48$ & $6 / 36$ & Amblyopia & Traumatic aphakia \\
79 & $6 / 24$ & $6 / 24$ & Epiretinal membrane & IOL disclocation \\
82 & $6 / 36$ & $6 / 24$ & Amblyopia & IOL dislocation \\
86 & $6 / 36$ & $6 / 24$ & None & Complicated phaco \\
\hline
\end{tabular}

In our series, $66.6 \%$ of patients achieved a final acuity better than that recorded pre-operatively. However, not every patient underwent measurement of their best-corrected visual acuity (BCVA) with pre-operative spectacle refraction. This was particularly the case in patients without ocular comorbidity who required Artisan implantation following complicated phacoemulsification surgery. Thus, the percentage of patients improving on their pre-operative best-recorded visual acuity may be artificially elevated. Nevertheless, the overall proportion of patients achieving final acuities of $6 / 12$ or better, $64.1 \%$ is highly encouraging.

If we consider the number of patients without preoperative ocular comorbidity, the proportion of patients achieving $6 / 12$ or better increases to $85.2 \%$, which is comparable to the $85 \%$ of patients achieving the same level of acuity in the National Cataract Surgery Survey (NCSS), ${ }^{2}$ although not quite as good as the $92 \%$ attaining that level as their final refracted acuity. Not all patients in this series underwent final refraction before discharge and it is possible that further improvement was achieved.

However, the group of patients with pre-operative ocular comorbidity fared considerably worse, with only $16.7 \%$ achieving an acuity better than or equal to $6 / 12$, compared with the $65 \%$ (77\% at final refracted acuity) in the NCSS. ${ }^{2}$ This low figure reflects the type of comorbidity as previously detailed and also clearly highlights the relationship between comorbidity and poor visual outcome.

One particular concern with all types of anterior chamber lens is a reduction in corneal endothelial cell density. In this series, specular microscopy and corneal thickness measurements were not routinely performed and we are unable to comment on any such changes.

However, some studies have demonstrated a reduction in cell density. One series was performed on nine eyes with traumatic lens subluxation and showed reductions in density of $15 \%$ at 1 month and $14 \%$ at 12 months post-operatively. ${ }^{3}$ Another study reported on 26 eyes where Artisan phakic lens implantation was performed for moderate to high myopia, with a reduction in cell density of $14.05 \%$ after 5 years. ${ }^{4}$ A third study of 67 eyes with phakic lens implantation reported a density reduction of $10.9 \%$ at 3 years. ${ }^{5}$ As the latter two studies looked at phakic lens implants, the data is not directly comparable.

Most studies found by the authors suggested stability of endothelial cell counts, although again these were reporting findings in phakic eyes undergoing refractive surgery; those with reasonably large numbers of enrolled patients (155 and 90 eyes) did not demonstrate any significant loss of cell density. ${ }^{6,7}$ Another study reported stabilisation to physiologically normal levels at 3 years in 129 eyes. ${ }^{8}$ The longest follow-up period of any series was 9.5 years in 3 children who underwent Artisan lens implantation following congenital cataract extraction, and reported no difference in cell count when compared to the unoperated eye. ${ }^{9}$

Other potential complications following Artisan aphakia lens implantation include uveitis, glaucoma, hyphaema, and cystoid macular oedema. Although there have been some case reports of such developments, ${ }^{10,11}$ including a case of pupillary block glaucoma following a phakic implantation, ${ }^{12}$ no large series have reported a significant incidence of these, and we have not encountered such complications to date. In our series, a surgical PI should preclude the possibility of pupillary block.

There are alternatives to Artisan aphakia IOL placement, including angle-fixated AC IOLs and scleral-fixated posterior chamber IOLs (SPCIOLs). Complications following angle placement of AC IOLs include corneal decompensation, uveitis, secondary 
glaucoma, distorted pupil, cystoid macular oedema, and retinal detachment. ${ }^{13}$

Yang et $\mathrm{ll}^{14}$ reported a series of 65 eyes in 61 patients who underwent SPCIOL placement, with $66 \%$ of the patients achieving improved or unchanged BCVA at the final follow-up. In total, $37 \%$ of patients experienced preor post-operative adverse events. Their methodology and patient cohort (non-vitrectomised eyes) were sufficiently different from ours to prevent a direct comparison of outcomes, but placement of SPCIOLs requires more surgical manipulation and carries the risk of vitreous traction with the possibility of ensuing rhegmatogenous retinal detachment. ${ }^{15}$ Other possible complications include tilting of the implant, decentration, or dislocation, and transscleral suture erosion.

\section{Conclusion}

This study shows that Artisan aphakia lens implantation is capable of delivering good visual outcomes with a low complication rate in patients who are unable to undergo intracapsular or sulcus IOL positioning. A prospective study, including serial endothelial cell density measurement, morphology, and corneal thickness would provide further useful information on the long-term safety in pseudophakic patients.

\section{Summary}

What was known before

- Artisan/Verisyse lenses have given good outcomes in traumatic aphakia.

\section{What this study adds}

- Good visual outcomes are also achievable in other causes of aphakia.

- Outcomes compare favourably with scleral-fixated lenses.

\section{Conflict of interest}

The authors declare no conflict of interest.

\section{References}

1 Apple DJ, Brems RN, Park RB, Norman DK, Hansen SO, Tetz MR et al. Anterior chamber lenses. Part 1: complications and pathology and a review of designs. J Cataract Refract Surg 1987; 13: 157-174.
2 Desai P, Minassian DC, Reidy A. National cataract surgery survey 1997-98: a report of the results of the clinical outcomes. Br J Ophthalmol 1999; 83: 1336-1340.

3 Kodjikian L, Beby F, Spire M, Gambrelle J, Hubert I, Burillon $\mathrm{C}$ et al. Combined pars plana phacofragmentation, vitrectomy, and Artisan lens implantation for traumatic subluxated cataracts. Retina 2006; 26(8): 909-916.

4 Silva RA, Jain A, Manche EE. Prospective long-term evaluation of the efficacy, safety, and stability of the phakic intraocular lens for high myopia. Arch Ophthalmol 2008; 126(6): 775-781.

5 Landesz M, Worst JG, van Rij G. Long-term results of correction of high myopia with an iris claw phakic intraocular lens. J Refract Surgery 2000; 16(3): 310-316.

6 Maloney RK, Nguyen LH, John ME. Artisan phakic intraocular lens for myopia: short-term results of a prospective, multicenter study. Ophthalmology 2002; 109(9): 1631-1641.

7 El Danasoury MA, El Maghraby A, Gamali TO. Comparison of iris-fixed Artisan lens implantation with excimer laser in situ keratomileusis in correcting myopia between -9.00 and -19.50 diopters: a randomized study. Ophthalmology 2002; 109(5): 955-964.

8 Budo C, Hessloehl JC, Izak M, Luyten GP, Menezo JL, Sener BA et al. Multicenter study of the Artisan phakic intraocular lens. J Cataract Refract Surg 2000; 26(8): 1163-1171.

9 Odenthal MTP, Sminia ML, Prick LJ, Gortzak-Moorstein N, Volker-Dieben HJ. Long-term follow-up of the corneal endothelium after artisan lens implantation for unilateral traumatic and unilateral congenital cataract in children: two case series. Cornea 2006; 25(10): 1173-1177.

10 Sridhar MS, Majji AB, Vaddavalli PK. Severe inflammation following iris fixated anterior chamber phakic intraocular lens for myopia. Eye 2006; 20: 1094-1095.

11 Kodjikian L, Beby F, Spire M, Gambrelle J, Hubert I, Burillon $\mathrm{C}$ et al. Combined pars plana phacofragmentation, vitrectomy, and Artisan lens implantation for traumatic subluxated cataracts. Retina 2006; 26(8): 909-916.

12 Hogewind BF, Theelen T. Slit-lamp-adapted optical coherence tomography of pupillary block after Artisan lens implantation for aphakia. Int Ophthalmol 2007; 27(5): 337-338.

13 Hennig A, Johnson GJ, Evans JR, Lagnado R, Poulson A, Pradhan $\mathrm{D}$ et al. Long term clinical outcome of a randomised controlled trial of anterior chamber lenses after high volume intracapsular cataract surgery. Br J Ophthalmol 2001; 85(1): 11-17.

14 Yang YF, Bunce C, Dart JKG, Johnston RL, Charteris DG. Scleral-fixated posterior chamber intraocular lenses in non vitrectomised eyes. Eye 2006; 20: 64-70.

15 Tsunoda K, Migita M, Nakashizuka T, Kohzuka T. Treatment of anterior vitreous before suturing an intraocular lens to the ciliary sulcus. J Cataract Refract Surg 1996; 22: 222-226. 